\title{
Minimal measurements of the gate fidelity of a qudit map
}

\author{
E. Bagan, M. Baig, and R. Muñoz-Tapia \\ Grup de Física Teòrica and IFAE, Facultat de Ciències, Edifici Cn, Universitat Autònoma de Barcelona, \\ 08193 Bellaterra (Barcelona), Spain
}

(Received 6 August 2002; published 23 January 2003)

\begin{abstract}
We obtain a simple formula for the average gate fidelity of a linear map acting on qudits. It is given in terms of minimal sets of pure state preparations alone, which may be interesting from the experimental point of view. These preparations can be seen as the outcomes of certain minimal positive operator valued measures. The connection of our results with these generalized measurements is briefly discussed.
\end{abstract}

DOI: 10.1103/PhysRevA.67.014303

PACS number(s): 03.67.-a, 89.70.+c, 03.65.Wj

The interaction with the environment has a significant negative impact on any physical implementation of a unitary gate or a quantum channel. Decoherence, turning pure states into mixed states, creeps up and unitarity is lost in the Hilbert subspace of the signal states. The characterization of the quality of such implementations is, hence, of utmost importance for quantum computation from both experimental and theoretical points of view [1].

A physical quantum gate or channel is best described by a trace-preserving linear map or superoperator $\mathcal{E}$, which is assumed to be an approximation of a unitary operator $U$ characterizing the quantum gate ( $U=1$ for a quantum channel). The average gate fidelity

$$
\bar{F}(\mathcal{E}, U)=\int d \psi \operatorname{tr}\left[U \rho_{\psi} U^{\dagger} \mathcal{E}\left(\rho_{\psi}\right)\right]
$$

where $d \psi$ is the invariant Haar measure on the space of pure states $|\psi\rangle$ (i.e., $d \psi=d \psi^{\prime}$ if $\left|\psi^{\prime}\right\rangle=U|\psi\rangle$ ) and $\rho_{\psi}=|\psi\rangle\langle\psi|$, is recognized as a convenient figure of merit and is widely used to assess the quality of a quantum gate or channel.

For gates acting on qubits, Bowdrey et al. [2] have recently derived a simple formula for $\bar{F}(\mathcal{E}, U)$, from which they obtain a convenient way of actually measuring the gate fidelity in a laboratory. It amounts to replacing the integral in Eq. (1) by a sum over a finite number of pure states whose Bloch vectors point at the vertices of an octahedron or a tetrahedron inscribed in the Bloch sphere. Nielsen [3] has obtained a similar formula for qudits (i.e., quantum states belonging to a $d$-dimensional Hilbert space) in terms of a finite set of unitary operations $U_{j}$ orthogonal with respect to the Hilbert-Schmidt inner product, namely, such that $\operatorname{tr}\left(U_{i}^{\dagger} U_{j}\right)=d \delta_{i j}$. His derivation is based on Horodeckis' formula connecting $\bar{F}(\mathcal{E}, 1) \equiv \bar{F}(\mathcal{E})$ with the entanglement fidelity [4], for which he also provides a simple proof. Our aim is to obtain a more natural expression of the average gate fidelity so that only measurements over a (minimum) number of pure states need to be performed to verify $\bar{F}$ experimentally. Interestingly enough, the solution to this problem is not far removed from that of obtaining minimal positive operator valued measurements (POVMs) in the context of optimal communication of directions through a quantum (spin) channel, which has received much attention over the last few years [5-7].
In the first part of this paper we derive a general expression for $\bar{F}$ in terms of the $\mathrm{SU}(d)$ group generators. In the second part we write $\bar{F}$ as an average of measurements over a finite and minimal number of pure state preparations, which may be experimentally relevant.

We first notice that due to the invariance of $d \psi$ one has $\bar{F}(\mathcal{E}, U)=\bar{F}\left(\mathcal{E}^{\prime}, 1\right)=\bar{F}\left(\mathcal{E}^{\prime}\right)$, where $\mathcal{E}^{\prime}(\rho) \equiv \mathcal{E}\left(U^{\dagger} \rho U\right)$. Hence we only need to consider the simpler form $\bar{F}(\mathcal{E})$ without any loss of generality. Furthermore, the uniform measure $d \psi$ can be effectively realized in the following way: $\int d \psi \mathcal{F}(\psi)$ $\equiv \int d U \mathcal{F}\left(U \psi_{0}\right)$, where $\mathcal{F}$ is any function of $\rho_{\psi},\left|\psi_{0}\right\rangle$ is a fixed reference state, and $d U$ is the Haar measure of $\mathrm{SU}(d)$ normalized such that $\int d U=1$. This ensures that $\int d \psi \mathcal{F}(\psi)$ $=\int d \psi \mathcal{F}\left(U^{\prime} \psi\right)$ for any $U^{\prime} \in \mathrm{SU}(d)$. Obviously, not all the $d^{2}-1$ parameters involved in $d U$ are physically significant. For example, for qubits $(d=2), d \psi=d n$ is the uniform measure on the 2-sphere $S^{2}$, which can be parametrized by the Euler angles $\alpha$ and $\beta$. If $\left|\psi_{0}\right\rangle$ is an eigenstate of the Pauli matrix $\sigma_{z}$, any function of $\rho_{\psi}$ is independent of the third Euler angle $\gamma$. Hence, $\int d \psi \mathcal{F}=\left[\int d \gamma /(2 \pi)=1\right] \times \int d n \mathcal{F}$ $=\int d U \mathcal{F}$. For qudits, $d n$ must be replaced by the invariant measure of $\mathrm{SU}(d) /[\mathrm{SU}(d-1) \times \mathrm{U}(1)]$, since any reference density matrix $\rho_{0} \equiv\left|\psi_{0}\right\rangle\left\langle\psi_{0}\right|$ is now invariant under $\mathrm{SU}(d$ $-1) \times U(1)$. Note that the number of parameters match, as qudits depend on $2(d-1)$ real variables. With all this, we finally have

$$
\bar{F}(\mathcal{E})=\int d U \operatorname{tr}\left[U \rho_{0} U^{\dagger} \mathcal{E}\left(U \rho_{0} U^{\dagger}\right)\right]
$$

There exists a $\left(d^{2}-1\right)$-dimensional unitary vector with components $n_{0}^{a}, a=1,2, \ldots, d^{2}-1$, such that the density matrix $\rho_{0}$ can be written as

$$
\rho_{0}=\frac{1}{d}+k_{d} n_{0}^{a} T_{a} \equiv \frac{1}{d}+k_{d} \vec{n}_{0} \cdot \vec{T},
$$

where $k_{d}=\sqrt{2(d-1) / d}$ and $\left\{T_{a}\right\}$ are the (Hermitian and traceless) generators of $\mathrm{SU}(d)$ [8], normalized so that $\operatorname{tr}\left(T_{a} T_{b}\right)=\delta_{a b} / 2$. They can be chosen as $T_{a}=\lambda_{a} / 2$, where $\lambda_{a}$ are a generalization of the Gell-Mann matrices of SU(3). Throughout this paper a sum over repeated latin indices is understood. It is straightforward to obtain 


$$
\vec{n}_{0}=\frac{2}{k_{d}} \operatorname{tr}\left(\rho_{0} \vec{T}\right) .
$$

We now recall the well-known relation [8]

$$
U T_{a} U^{\dagger}=(\mathcal{A} U)_{a}^{b} T_{b},
$$

where $\mathcal{A}$ stands for the adjoint representation of $\mathrm{SU}(d)$. We also recall the orthogonality of the irreducible representations of compact groups, which in the present case implies

$$
\begin{gathered}
\int d U(\mathcal{A U})_{a}{ }^{b}=0 \\
\int d U(\mathcal{A} U)_{a}{ }^{b}(\mathcal{A} U)_{c}{ }^{d}=\frac{\delta_{a c} \delta^{b d}}{d^{2}-1} .
\end{gathered}
$$

Assuming that $\mathcal{E}$ is linear and trace preserving, one gets

$$
\bar{F}(\mathcal{E})=\frac{1}{d}+\frac{2}{d(d+1)} \sum_{a=1}^{d^{2}-1} \operatorname{tr}\left[T_{a} \mathcal{E}\left(T_{a}\right)\right] .
$$

This is the generalization of the formula for qubits

$$
\bar{F}(\mathcal{E})=\frac{1}{2}+\frac{1}{3} \sum_{i=x, y, z} \operatorname{tr}\left[\frac{\sigma_{i}}{2} \mathcal{E}\left(\frac{\sigma_{i}}{2}\right)\right]
$$

obtained in Ref. [2]. This concludes the first part of this paper.

The key ingredient of the derivation above is the orthogonality relation of the group representations, exemplified by Eqs. (6) and (7). We will show that it is possible to find a discrete version of these equations. Namely, one can find a finite set of $\mathrm{SU}(d)$ elements $\left\{U_{r}\right\}$ and positive constants $\left\{c_{r}\right\}$ such that

$$
\begin{gathered}
\sum_{r} c_{r}\left(\mathcal{A} U_{r}\right)_{a}{ }^{b}=0 \\
\sum_{r} c_{r}\left(\mathcal{A} U_{r}\right)_{a}{ }^{b}\left(\mathcal{A} U_{r}\right)_{c}{ }^{d}=\frac{\delta_{a c} \delta^{b d}}{d^{2}-1} .
\end{gathered}
$$

With this we can reverse the steps going from Eq. (2) to Eq. (8) using the relations (10) and (11) instead of their continuous versions (6) and (7), and obtain

$$
\bar{F}(\mathcal{E})=\sum_{r} c_{r} \operatorname{tr}\left[\rho_{r} \mathcal{E}\left(\rho_{r}\right)\right]
$$

This equation has a very convenient form that allows setting up experimental tests to determine the fidelity of a gate or channel, as will be discussed below.

Let us briefly discuss the solutions to Eqs. (10) and (11). The idea is to generalize the concept of a finite set of isotropically distributed unit vectors introduced in Ref. [6] and adapt it to the problem at hand. A sufficient condition for Eq. (12) to hold can be obtained by contracting Eqs. (10) and (11) with $n_{0}^{a}$. If we define

$$
n_{0}^{a}\left(\mathcal{A} U_{r}\right)_{a}^{b} \equiv n_{r}^{b}
$$

conditions (10) and (11) lead to

$$
\sum_{r} c_{r} n_{r}^{b}=0 ; \quad \sum_{r} c_{r} n_{r}^{b} n_{r}^{d}=\frac{\delta^{b d}}{d^{2}-1} .
$$

In this sense, we may qualify the set $\left\{n_{r}\right\}$ as isotropically distributed [as far as the adjoint representation $\mathrm{SU}(d)$ is concerned]. We have traded the problem of finding $\left\{U_{r}\right\}$ for that of finding $\left\{n_{r}\right\}$. Note, however, that the set of matrices $\{\mathcal{A} U\}$ is a proper subgroup of $\mathrm{SO}\left(d^{2}-1\right)$. Hence, no vector on the sphere $\mathrm{S}^{d^{2}-2}$ is admissible. We will come back to this issue below. The relations (10), (11), and (14) also appear in a rather different context: the construction of finite positive operator valued measurements that are optimal for communicating a direction $[6,7,9]$. Leaving aside an overall trivial normalization (from Eq. (14) it follows that $\Sigma_{r} c_{r}=1$, whereas in Ref. [9] $\Sigma_{r} c_{r}=d$ ), the results in those papers can be readily used here. In particular, it is proved in Ref. [9] that solutions of Eq. (14) exist and the minimal one is given by a set of $d^{2}$ vectors pointing at the vertices of a regular hypertetrahedron or, more properly, $\left(d^{2}-1\right)$ simplex, inscribed on $\mathrm{S}^{d^{2}-2}$. This hypertetrahedron is defined by the condition

$$
\vec{n}_{r} \cdot \vec{n}_{s}=-\frac{1}{d^{2}-1}, \quad r \neq s
$$

and the exact overall orientation has to be chosen so that all vectors $\vec{n}_{r}$ are of the form (13). For this hypertetrahedron all the coefficients $c_{r}$ are equal: in our notation $c_{r}=1 / d^{2}, r$ $=1,2, \ldots, d^{2}$. An explicit form of $\vec{n}_{r}$ for $\mathrm{SU}(3)$ can be found in Ref. [9], where also the general case is briefly discussed. The solution is more conveniently expressed in terms of states $\left|\psi_{r}\right\rangle$ such that

$$
\left|\psi_{r}\right\rangle\left\langle\psi_{r}\right| \equiv \rho_{r}=U_{r} \rho_{0} U_{r}^{\dagger}=\frac{1}{d}+k_{d} \vec{n}_{r} \cdot \vec{T}
$$

Then Eq. (15) translates into

$$
\left|\left\langle\psi_{r} \mid \psi_{s}\right\rangle\right|^{2}=\frac{1}{d+1}, \quad r \neq s
$$

Since this equation is a condition on states, Eq. (13) is automatically satisfied by the corresponding Bloch vectors.

For SU(3) a solution of Eq. (17) has the simple form

$$
\left|\psi_{r}\right\rangle=\frac{1}{\sqrt{2}}\left(\begin{array}{c}
1 \\
e^{2(r-1) \pi i / 3} \\
0
\end{array}\right), \quad r=1,2,3 .
$$

The remaining six states are obtained by applying cyclic permutations to the components of $\left|\psi_{1-3}\right\rangle$ (a different choice of states is given in Ref. [9]). From the experimental point of view, the states (18) have a very appealing form; each of them involves a linear combination of only two states of the 
computational basis. If the qutrit is implemented by say three atomic levels, only two levels need to be manipulated to prepare each one of the $\rho_{r}$.

In the $\mathrm{SU}(2)$ case, a solution in terms of $\vec{n}_{r}$ is more simple and transparent, mainly because $\mathrm{SU}(2)$ is isomorphic to $\mathrm{SO}(3)$, which makes Eq. (13) trivially satisfied by any vector of $S^{2}$. A compact solution is given by

$$
\vec{n}_{1}=\frac{1}{\sqrt{3}}(1,1,1), \quad \vec{n}_{2}=\frac{1}{\sqrt{3}}(-1,-1,1),
$$

and $\vec{n}_{3}, \vec{n}_{4}$ are again obtained by applying cyclic permutations to the components of $\vec{n}_{2}$.

For the hypertetrahedra discussed above, Eq. (12) can be cast as

$$
\bar{F}(\mathcal{E})=\frac{1}{d^{2}} \sum_{r=1}^{d^{2}} \operatorname{tr}\left[\rho_{r} \mathcal{E}\left(\rho_{r}\right)\right]
$$

which is the $\mathrm{SU}(d)$ generalization of Bowdrey et al. $\mathrm{SU}(2)$ formula. This equation is our main result and provides a remarkably simple procedure for measuring $\bar{F}(\mathcal{E})$. One just has to average the fidelities for $d^{2}$ isotropically distributed pure states. Notice that all state preparations $\rho_{r}$ have the same weight in the average, thus reducing systematic errors. In some sense, Eq. (20) can be regarded as the average survival rate of the states $\left\{\rho_{r}\right\}$ in a quantum channel characterized by the linear map $\mathcal{E}$. There is an alternative way of writing Eq. (20), which may provide further insight. It is straightforward to verify that the projectors $O_{r} \equiv \rho_{r} / d$ are the complete set of positive operators (i.e., $\Sigma O_{r}=1$ ) of a minimal POVM. Thus, there exists a device characterized by $\left\{O_{r}\right\}$ whose preparations are precisely $\rho_{r}$. We can write Eq. (20) as

$$
\bar{F}(\mathcal{E})=\frac{1}{d} \sum_{r=1}^{d^{2}} \operatorname{tr}\left[O_{r} \mathcal{E}\left(\rho_{r}\right)\right]
$$

We readily see that the same device $\left\{O_{r}\right\}$ can be used for the preparations of the pure states $\left\{\rho_{r}\right\}$ as well as for the measurements over $\left\{\mathcal{E}\left(\rho_{r}\right)\right\}$.

Although we have presented the results for the minimal sets of $\rho_{r}$, for practical reasons, one may wish to use a larger number of states. This possibility is easily implemented in this framework, as conditions (13) and (14) are not specific of minimal sets but entirely general. For example, for qubits, one can find a set of six states whose Bloch vectors point at the vertices of a regular octahedron $[2,5,7])$. In this case there is a simple setting for preparing the states, just three Stern-Gerlach's oriented along the three orthogonal directions.

We are grateful to A. Acín and A. Bramon for helpful conversations. We acknowledge financial support from CIRIT Project No. SGR-00185, Spanish Ministry of Science and Technology Project No. BFM2002-02588, and the European Funds for Regional Development (FEDER).
[1] J.F. Poyatos, J.I. Cirac, and P. Zoller, Phys. Rev. Lett. 78, 390 (1995).

[2] M.D. Bowdrey et al., Phys. Lett. A 294, 258 (2002).

[3] M.A. Nielsen, Phys. Lett. A 303, 249 (2002).

[4] M. Horodecki, P. Horodecki, and R. Horodecki, Phys. Rev. A 60, 1888 (1999).

[5] S. Massar and S. Popescu, Phys. Rev. Lett. 74, 1259 (1995); N. Gisin and S. Popescu, ibid. 83, 432 (1999); E. Bagan et al., ibid. 85, 5230 (2000); A. Peres and P.F. Scudo, ibid. 86, 4160 (2001).
[6] E. Bagan, M. Baig, and R. Muñoz-Tapia, Phys. Rev. A 64, 022305 (2001).

[7] J.I. Latorre, P. Pascual, and R. Tarrach, Phys. Rev. Lett. 81, 1351 (1998).

[8] An introduction to group theory that closely follows our notation can be found in J.F. Cornwell, Group Theory in Physics (Academic Press, London, 1984).

[9] A. Acín, J.I. Latorre, and P. Pascual, Phys. Rev. A 61, 022113 (2000). 